\title{
Evaluation of substance $P$ as a neurotransmitter in equine jejunum
}

\author{
Erin D. Malone, DVM, PhD; Mathur S. Kannan, BVSc, PhD; David R. Brown, PhD
}

Objective-To determine whether substance P (SP)

Sample Population-Samples of jejunum juntained from horses that did not have lesions in the gastrointestinal tract.

Procedure-Jejunal smooth muscle strips, oriented in the plane of the circular or longitudinal muscle, were suspended isometrically in muscle baths. Neurotransmitter release was induced by electrical field stimulation (EFS) delivered at 2 intensities (30 and $70 \mathrm{~V}$ ) and various frequencies on muscle strips that were maintained at low tension or were under contraction. A neurokinin-1 receptor blocker (CP$96,345)$ was added to baths prior to EFS to interrupt SP neurotransmission. Additionally, direct effects of $\mathrm{SP}$ on muscle strips were evaluated, and SP-like immunoreactivity was localized in intestinal tissues, using indirect immunofluorescence testing.

Results - Substance P contracted circularly and longitudinally oriented muscle strips. Prior treatment with CP-96,345 altered muscle responses to SP and EFS, suggesting that SP was released from depolarized myenteric neurons. Depending on orientation of muscle strips and stimulation variables used, CP-96,345 increased or decreased the contractile response to EFS. Substance P-like immunoreactivity was detected in the myenteric plexus and circular muscle layers.

Conclusions and Clinical Relevance-Substance $P$ appears to function as a neurotransmitter in equine jejunum. It apparently modulates smooth muscle contractility, depending on preexisting conditions. Effects of SP may be altered in some forms of intestinal dysfunction. Altering SP neurotransmission in the jejunum may provide a therapeutic option for motility disorders of horses that are unresponsive to adrenergic and cholinergic drugs. (Am J Vet Res 2000;61: 1178-1184)

$\mathrm{N}$ onadrenergic-noncholinergic (NANC) neurotransmission recently has been documented in the equine jejunum. ${ }^{1}$ In other species, it has been documented that substance $\mathbf{P}$ (SP) functions as a NANC neurotransmitter. ${ }^{2}$ Substance $\mathrm{P}$ is an undecapeptide that belongs to the tachykinin family and acts preferentially on G-protein-coupled neurokinin (NK-1) receptors. Substance $\mathrm{P}$ has a wide range of action, producing smooth muscle contraction in the respiratory, gastrointestinal, and urogenital tracts, stimulating vasodilation and glandular secretions, and influencing pain, inflammation, and immune responses. ${ }^{3}$ Within the gastrointestinal tract specifically, SP increases peristalsis, ion

Received Jul 30, 1999.

Accepted Dec 1, 1999.

From the Departments of Clinical and Population Sciences (Malone) and Veterinary PathoBiology (Kannan, Brown), College of Veterinary Medicine, University of Minnesota, St Paul, MN 55108. secretion, and blood flow while inhibiting gastric acid secretion and intestinal absorption. ${ }^{4,5}$ Substance $\mathrm{P}$ plays a role in the ascending-contraction component of the peristaltic reflex in guinea pigs and humans, ${ }^{6,7}$ appears to be involved in the colonoileal reflex of ileocecal sphincter contraction in response to distention of the proximal portion of the colon in cats, ${ }^{8}$ and is involved in release of acetylcholine (Ach) for the muscarinic inhibitory brake reflex of the small intestines in dogs. ${ }^{9}$

Substance P-like immunoreactivity has been detected throughout the equine intestinal tract. ${ }^{10-14}$ Neuronal cell bodies immunoreactive for SP have been identified in the myenteric and submucosal plexuses, with nerve fibers projecting to the circular muscle and to the mucosa. ${ }^{11,13}$ Substance $P$ also stimulates in vivo contractility of the colon ${ }^{15}$ and in vitro contractility of the small intestine and colon of horses. ${ }^{16}$ Finally, the NK-1 receptor has been identified in the pelvic flexure and other portions of the equine gastrointestinal tract. ${ }^{16,17}$

Increased SP concentrations and NK-1-receptor binding sites have been documented in the colon of humans with ulcerative colitis and granulomatous inflammation (Crohn's disease), and decreased concentrations of SP have been found in patients with chronic severe constipation. ${ }^{18,19}$ Concentrations of SP are altered in laboratory animals during intestinal inflammation, and the neuropeptide may be involved in subsequent changes in motility or in the local immune response. ${ }^{20,21}$ In horses, Moore et $\mathrm{al}^{22}$ documented a pattern toward increased concentrations of SP in colonic mesenteric arteries during early reperfusion in a low-flow colonic ischemia model. In horses affected by dysautonomia (grass sickness), extensive degranulation of peptidergic fibers with decreased amounts of SP-like immunoreactivity were detected and appeared to be an early event in the course of the disease. ${ }^{10}$ Reduced concentrations of SP in sensory neurons may account for the lack of severe signs of pain with this disease, despite substantial distention of the small intestines. ${ }^{2}$ Sonea et $\mathrm{al}^{17}$ were unable to detect an alteration in tachykinin-receptor binding in the pelvic flexure after a $720^{\circ}$ torsion was imposed for 60 minutes. Continued detection of these receptors despite severe metabolic changes may provide a new therapeutic option for modifying motility of the gastrointestinal tract.

Although the aforementioned studies provided suggestive evidence for the activity of SP within the gastrointestinal tract, it must be proven that SP is released from myenteric neurons for it to be classified as an enteric neurotransmitter in horses. ${ }^{2}$ The objective of the study reported here was to document release of SP from jejunal myenteric neurons depolarized in vitro by use of electrical field stimulation (EFS). Previously, 
we have identified jejunal NANC intrinsic neurotransmission with relaxant and contractile effects. We hypothesized that SP was responsible for a portion of the NANC contractile component. To further support our hypothesis, we anticipated that the jejunum would respond to SP in a similar manner to the response for neuronal stimulation and that evidence for SP in the myenteric plexus would be detectable by use of histochemical analysis.

\section{Materials and Methods}

Preparation of tissues-Intestinal tissues were obtained from horses without gastrointestinal tract disorders or systemic disease after they were anesthetized or within 30 minutes after they were euthanatized by IV administration of an overdose of pentobarbital. A segment of the middle portion of the jejunum was excised, cut along the mesenteric border, and placed in ice-cold oxygenated Krebs-bicarbonate buffer $\left(143 \mathrm{mM} \mathrm{Na}^{+}, 5.9 \mathrm{mM} \mathrm{K}^{+}, 134.1 \mathrm{mM} \mathrm{Cl}^{-}, 2.5 \mathrm{mM} \mathrm{Ca}^{2+}, 1.2\right.$ $\mathrm{mM} \mathrm{Mg}^{2+}, 21.0 \mathrm{mM} \mathrm{HCO}_{3}{ }^{-}, 1.2 \mathrm{mM} \mathrm{H}_{2} \mathrm{PO}_{4}{ }^{2-}$, and $10 \mathrm{mM}$ dextrose, $\mathrm{pH}$ 7.4). The mucosa and submucosa were removed, using sharp dissection. The remaining section of the intestine was cut into $2 \times 10$-mm strips that were oriented in the plane of the longitudinal or circular smooth muscle. Tissues were allowed to equilibrate for 60 minutes or until a consistent response to electrical field stimulation (EFS) was obtained.

Electrical field stimulation-Strips of smooth muscle were suspended isometrically under 2 to $4 \mathrm{~g}$ of tension (optimum strip tension) between bipolar platinum ring electrodes that were connected to a stimulator. ${ }^{\mathrm{b}}$ On the basis of results of preliminary studies, strips were subjected to EFS at intensities of 30 and $70 \mathrm{~V}$ and frequencies of $2,4,8$, and $15 \mathrm{~Hz}$ (pulse duration, 1 millisecond; pulse train, 10 seconds). These variables were chosen to depolarize nerves rather than muscle in equine intestine. ${ }^{c}$ A resting period of 60 seconds was allowed between each EFS episode for experiments at low (baseline) tension. The EFS also was applied to strips already contracted by use of histamine (10 to $100 \mu \mathrm{M})$, barium chloride $(100 \mu \mathrm{M})$, or carbachol $(10 \mu \mathrm{M})$. Because of the waning of the contraction amplitude, only 15 seconds were allowed between each EFS episode for strips that were already contracted. A maximum of 2 experiments at each tension (baseline and already contracted) were performed for each muscle strip to avoid fatigue. The order of addition of drugs was randomized throughout experiments to minimize changes attributable to fatigue of the muscle strips. In preliminary studies, $1 \mu \mathrm{M}$ indomethacin was added to the baths to determine whether there was evidence of intrinsic or induced prostaglandin release. Alterations were not detected, and indomethacin was not included in these studies.

Response to substance $\mathrm{P}$-To assess response of jejunal muscle to SP, a concentration-response curve was constructed with and without addition of a nonpeptide neurokinin-1 receptor blocker, CP-96,345. ${ }^{\mathrm{d}}$ Concentrations of SP were added ranging from $0.1 \mathrm{pM}$ to $1 \mu \mathrm{M}$. When used, $1 \mu \mathrm{M}$ CP96,345 was added $\geq 5$ minutes before the addition of SP.

Evaluation of SP release from neurons-To evaluate whether SP was released from depolarized myenteric neurons and functioned as a NANC neurotransmitter, CP-96,345 (0.1 to $1 \mu \mathrm{M}$ ) was used alone and in combination with $1 \mu \mathrm{M}$ atropine. The CP-96,345 was incubated for 5 to 10 minutes. Ability of CP-96,345 to inhibit the action of SP in equine intestine was confirmed prior to EFS studies. Substance P (10 to $100 \mu \mathrm{M}$ ) was added in an attempt to reverse the action of CP-96,345 on longitudinal muscle. Attempts at reversal were performed to determine specificity of drug effects.
Fixation and sectioning for immunohistochemical analysis-Tissues were collected from 20 clinically normal horses (18 adults and 2 fetuses) immediately after they were euthanatized for unrelated reasons. Sections of small intestine were opened and pinned flat on silicon-coated plates. Tissues from each horse were fixed in Zamboni solution (picric acid), plain formalin, or neutral-buffered formalin for 2 hours. ${ }^{23}$ Following fixation, tissues were washed in a series of decreasing concentrations of ethanol, followed by 4 washes in sodium-phosphate buffer. To decrease background fluorescence, tissues were incubated in $0.1 \%$ sodium cyanoborohydride for 30 minutes, followed by 3 washes in sodiumphosphate buffer. At least 24 hours prior to sectioning, tissues were placed in a $20 \%$ solution of sucrose at 4 C for cryoprotection. Alternatively, tissues were pinned flat on a wood base and frozen without fixation in a container of dry ice. Unfixed tissues were stored at $-70 \mathrm{C}$.

Small sections of tissue were placed in embedding medium and frozen at $-70 \mathrm{C}$. Two sections, cut in perpendicular planes, were embedded together. After $\geq 1$ hour of cooling, sections were cut on a cryostat $(-25 \mathrm{C})$ to a thickness of 15 $\mu \mathrm{m}$. Three pairs of tissue sections were mounted on each gelcoated slide. Prepared slides were stored at $-70 \mathrm{C}$.

Immunohistochemical staining-Stored slides were washed in 10\% casein for 1 hour and rinsed twice in PBS solution. All antibodies were diluted in PBS solution containing $0.3 \%$ triton, $1 \%$ bovine serum albumin, $2 \%$ serum (goat or rabbit), and $0.05 \%$ sodium azide. Tissues initially were absorbed with $10 \%$ serum (of the same species as the secondary antibody) for 1 hour at $4 \mathrm{C}$ to reduce background staining. Primary antibody was applied, and slides were incubated in a humidity chamber at $4 \mathrm{C}$ for 24 or 48 hours. After incubation, slides were rinsed 4 times in PBS solution. After application of a fluorochrome-labeled secondary antibody, slides were incubated in the dark at room temperature (25 C) for 1 hour. Slides again were washed 4 times in PBS solution and allowed to dry. Coverslips were applied using 90\% glycerol, and slides were examined using a fluorescent microscope.

Tissues were stained to detect glial protein (to outline neuronal tissue $e^{24}$, SP, and SP combined with choline acetyltransferase (ChAT) ${ }^{25}$ Primary antibody concentrations were as follows: rabbit anti-glial fibrillary acidic protein (GFAP; $1: 400),{ }^{\mathrm{e}}$ rabbit anti-SP $(1: 2,000),{ }^{\mathrm{f}}$ guinea pig anti-SP $(1: 300){ }^{\mathrm{g}}$ and rabbit anti-ChAT $(1: 2,000) .{ }^{\mathrm{h}}$ Goat-derived antibody to rat IgG labeled with the fluorochrome Cy3 (GAR-Cy3; [1:200]) or rabbit-derived anti-guinea pig IgGfluorescein isothiocyanate (FITC) conjugate (1:200) were used as the secondary antibodies. Slides were viewed by use of a fluorescent microscope (552 $\mathrm{nm}$ for $\mathrm{Cy} 3$ conjugates or $495 \mathrm{~nm}$ for FITC conjugates). Substitution of the primary antibody with nonimmune serum or PBS solution was used as a negative-control sample. Equine spinal cord was used as a positive-control sample for ChAT and SP staining. Specificity was evaluated for SP staining by absorbing the antibody with $15 \mu \mathrm{g}$ of $\mathrm{SP} / \mathrm{ml}$ and incubation for 1 hour at room temperature. In double-staining protocols, dilutions of antibodies were halved (eg, 1:200 instead of 1:400), and slides stained with only 1 antibody were used to compare slide quality.

Immunohistochemical evaluation-To identify SP-like and ChAT-like immunoreactivity, stained tissues were compared subjectively in a pairwise manner with control tissues from the same horse that had been fixed in the same manner. Six separate sections ( 3 of each orientation) from each horse were compared.

Statistical analyses-Alterations in muscle tension were evaluated for various concentrations of SP. Values for various concentrations of the reagents were compared, using a repeat- 
ed-measures ANOVA. Comparisons between SP and SP in the presence of CP-96,345 were made, using a 1-way ANOVA.

For EFS experiments, mean response to drugs in a minimum of 6 strips was calculated and analyzed for each drug or drug combination. Each comparison was performed only once in tissues from each horse. Various drugs from each class of inhibitors were used to determine interrelationships among neurotransmitters and to confirm unusual results. Results for EFS studies conducted at baseline tension were evaluated in terms of amplitude of contraction at the end of the EFS and 6 seconds after completion of EFS. Paired data were analyzed by use of the Wilcoxon signed-rank test for nonparametric data because of a lack of normal distribution in some data sets. Data obtained in studies of already-contracted strips were evaluated as a fraction of the initial amplitude at 3 times: 6 seconds after start of EFS (level A), at the end of EFS (level B; on response), and 6 seconds after end of EFS (level C; off response). These time points were chosen to reflect peak effects for strips at baseline tension and plateau responses for already-contracted muscle strips. A 1-way ANOVA was used to compare data. Comparisons also were made between stimulus intensities, pulse durations, and repetitions of EFS, using a 1-way ANOVA. Significance was defined at $P<0.05$ for all tests. Results that did not have significant effects were evaluated to determine the power of the test (inability to detect a true significant difference because of a low number of samples).

\section{Results}

Muscle response to substance $\mathrm{P}$-Longitudinal muscle strips had a concentration-dependent increase in tension in response to SP (median effective concen-

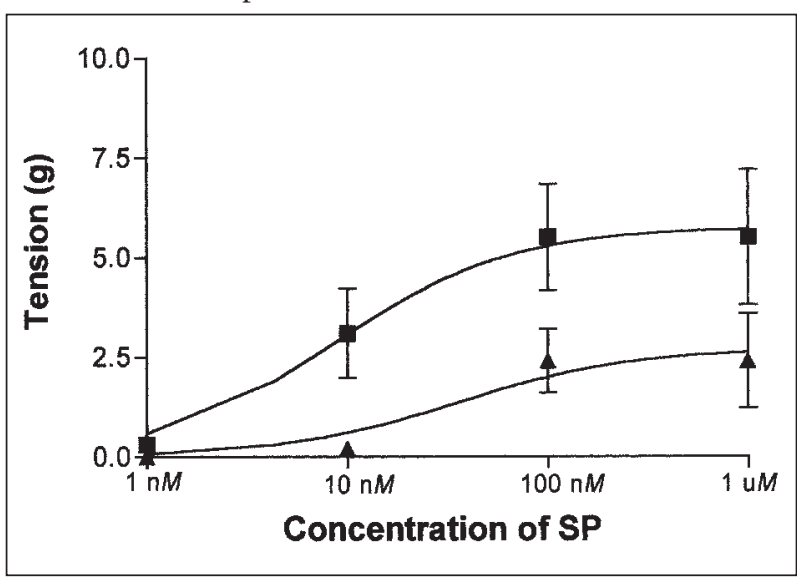

Figure 1-Concentration-response curve for the effect of various concentrations of substance P (SP; $\mathbf{\square})$ and SP and $1 \mu \mathrm{M} \mathrm{CP}$ 96,345 ( $\mathbf{\Delta}$ ) on tension of circularly oriented jejunal muscle. Notice the shift in the curve for muscle strips incubated with SP and $1 \mu \mathrm{M}$ CP-96,345. Values represent mean \pm SEM for $n \geq 7$ muscle strips obtained from the same number of horses. tration $\left.\left[\mathrm{EC}_{50}\right]=30 \mathrm{nM}\right)$. Generally, frequency of contraction was not altered, but amplitude of contraction decreased as tension increased. Circular muscle appeared to be more sensitive to the effects of SP $\left(E_{50}\right.$ of approx $8 \mathrm{nM})$. The SP receptor blocker noncompetitively inhibited the circular muscle response to SP, shifting the $\mathrm{SP} \mathrm{EC}_{50}$ to $0.1 \mu \mathrm{M}$ and decreasing its efficacy (Fig 1).

Substance P-related neurotransmission in longitudinal muscle-In strips at baseline tension (2 to 4 g), EFS at 30 and $70 \mathrm{~V}$ produced a frequency-dependent contractile response for the duration of the stimulation (Table 1). This contraction peaked at or near the end of the stimulation. Addition of CP-96,345 did not alter this response (ie, on response). When longitudinal muscle strips were already contracted, a biphasic on response to EFS was most common. ${ }^{1}$ Electrical field stimulation initially caused a decrease in the amount of tension (level A), which persisted during the first half of stimulation and was most consistent at the higher-intensity stimulation $(70 \mathrm{~V})$. This was followed by a second phase (level B), which consisted of a gradual increase in tension, generally returning the strips to the original degree of tension. Addition of CP96,345 caused a significant decrease in the contractile on response during EFS at $70 \mathrm{~V}$ during level B (ie, tissues remained relaxed) but did not alter the response to EFS at $30 \mathrm{~V}$. In uncontracted and already-contracted longitudinally oriented tissues, the on response was usually followed by a separate contractile response that began immediately after cessation of EFS (ie, the off response). Addition of CP-96,345 increased the contractile off response at both intensities and in alreadycontracted tissues. Substance P did not significantly reverse the action of CP-96,345 in longitudinal muscle.

Substance P-related neurotransmission in circular muscle-In circularly oriented muscle strips at baseline tension, tissues did not contract significantly during EFS at $30 \mathrm{~V}$ (Table 2). However, EFS at $70 \mathrm{~V}$ caused a contractile response that was peaking at the end of EFS. In circularly oriented muscle strips stimulated while under higher tension, the response pattern was similar to that of the longitudinally oriented muscle; however, the contractile and relaxant responses were generally more exaggerated (ie, deviated further from the original degree of tension). Addition of CP96,345 significantly inhibited the contractile response to EFS at $70 \mathrm{~V}$, but not at $30 \mathrm{~V}$, for level B (on response) in tissues at higher tension. In tissues at

Table 1-Response (mean \pm SEM) of longitudinally oriented muscle strips of equine jejunum to the neurokinin-1 receptor blocker CP96,345 after electrical field stimulation (EFS) at 30 and $70 \mathrm{~V}$

\begin{tabular}{|c|c|c|c|c|c|c|c|c|}
\hline \multirow[b]{2}{*}{ Variable } & \multicolumn{4}{|c|}{$30 \mathrm{~V}$} & \multicolumn{4}{|c|}{$70 \mathrm{~V}$} \\
\hline & $\mathbf{n}$ & Control & CP-96,345 & $P$ value & $\mathbf{n}$ & Control & CP-96,345 & $P$ value \\
\hline Baseline tension on response $^{*}(\mathrm{~g})$ & 21 & $8.62 \pm 1.34$ & $8.38 \pm 1.53$ & $0.77 \dagger$ & 21 & $11.44 \pm 1.36$ & $8.76 \pm 1.17$ & $0.30 t$ \\
\hline AC-A $\ddagger(\%)$ & 10 & $1.00 \pm 0.03$ & $0.92 \pm 0.07$ & $0.27 \dagger$ & 23 & $0.91 \pm 0.03$ & $0.84 \pm 0.05$ & $0.22 \dagger$ \\
\hline$A C-B \ddagger(\%)$ & 10 & $1.01 \pm 0.03$ & $0.97 \pm 0.07$ & $0.65 t$ & 23 & $1.06 \pm 0.03$ & $0.86 \pm 0.05$ & 0.05 \\
\hline Baseline tension off response ${ }^{*}(\mathrm{~g})$ & 21 & $5.10 \pm 1.16$ & $4.54 \pm 1.28$ & 0.69 & 21 & $8.51 \pm 1.49$ & $7.90 \pm 1.23$ & 0.45 \\
\hline AC-C off response $(\%)$ & 10 & $1.07 \pm 0.04$ & $1.26 \pm 0.08$ & 0.05 & 23 & $1.24 \pm 0.03$ & $1.42 \pm 0.05$ & 0.02 \\
\hline
\end{tabular}


Table 2-Response (mean \pm SEM) of circularly oriented muscle strips of equine jejunum to the NK-1 receptor blocker CP-96,345 after electrical field stimulation (EFS) at 30 and $70 \mathrm{~V}$

\begin{tabular}{|c|c|c|c|c|c|c|c|c|}
\hline \multirow[b]{2}{*}{ Variable } & \multicolumn{4}{|c|}{$30 \mathrm{~V}$} & \multicolumn{4}{|c|}{$70 \mathrm{~V}$} \\
\hline & $\mathbf{n}$ & Control & CP-96,345 & $P$ value & $\mathbf{n}$ & Control & CP-96,345 & $P$ value \\
\hline Baseline tension on response ${ }^{*}(\mathrm{~g})$ & 10 & $1.72 \pm 1.15$ & $0.64 \pm 0.35$ & $0.55 \dagger$ & 15 & $5.69 \pm 0.67$ & $3.93 \pm 0.67$ & $0.89 \dagger$ \\
\hline $\mathrm{AC}-\mathrm{A}(\%)$ & 10 & $0.50 \pm 0.05$ & $0.46 \pm 0.09$ & $0.74 \dagger$ & 10 & $0.49 \pm 0.04$ & $0.40 \pm 0.09$ & $0.32 \dagger$ \\
\hline$A C-B(\%)$ & 10 & $0.57 \pm 0.08$ & $0.68 \pm 0.13$ & 0.47 & 10 & $1.01 \pm 0.06$ & $0.71 \pm 0.13$ & 0.05 \\
\hline Baseline tension off response ${ }^{*}(\mathrm{~g})$ & 10 & $12.12 \pm 4.22$ & $12.92 \pm 2.51$ & $0.41 \dagger$ & 15 & $15.72 \pm 2.86$ & $14.32 \pm 1.76$ & $0.52 \dagger$ \\
\hline$A C-C$ off response $(\%)$ & 10 & $1.30 \pm 0.10$ & $1.36 \pm 0.18$ & 0.75 & 10 & $1.38 \pm 0.08$ & $1.40 \pm 0.18$ & 0.92 \\
\hline
\end{tabular}

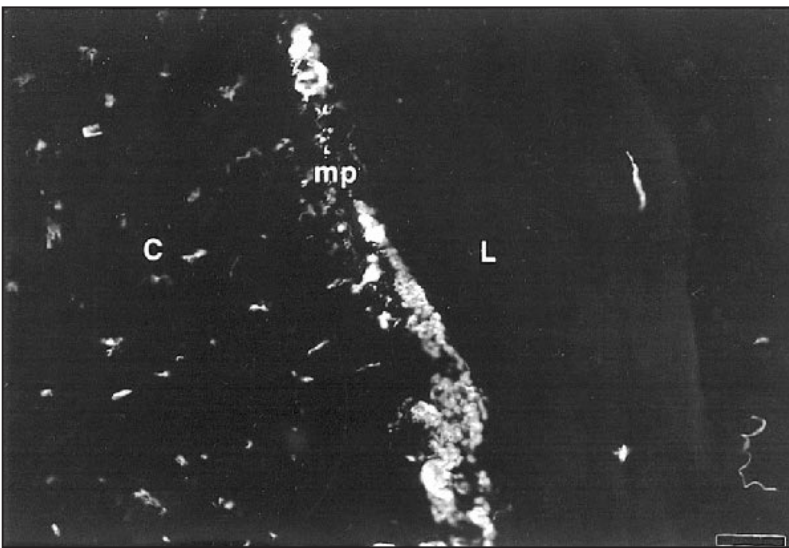

Figure 2-Photomicrograph of a section of jejunum stained with antibody to glial fibrillary acidic protein. Abundant staining is evident in the myenteric plexus ( $\mathrm{mp}$ ) and circular muscle (C). Minimal staining is evident in the longitudinal muscle (L). Tissues were fixed in Zamboni solution. Bar $=25 \mu \mathrm{m}$.

baseline tension, CP-96,345 caused a similar inhibition of the contractile on response to EFS at 30 and 70 $\mathrm{V}$, but the changes were not significantly different. However, the power of the tests ( $\beta$ error) was low because of high variability and a small number of samples. Similar to longitudinally oriented muscle strips exposed to EFS, a strong contractile response in circularly oriented muscle strip immediately followed cessation of EFS. For already-contracted tissues exposed to EFS at $30 \mathrm{~V}$ in the presence of atropine, the NK-1 receptor blocker significantly inhibited the off response at 2 and $4 \mathrm{~Hz}$, whereas it increased the contractile off response at 8 and $15 \mathrm{~Hz}$. A similar pattern was observed in tissues at baseline tension, but changes were not significantly different. Addition of CP-96,345 did not alter the off response of alreadycontracted strips stimulated at $70 \mathrm{~V}$.

General immunohistochemical analysis-Tissues fixed with normal-buffered formalin and Zamboni solution retained adequate immunogenicity for GFAP, ChAT, and SP staining; however, SP immunoreactivity (IR) was seemingly more intense after fixation with Zamboni solution, whereas ChAT-IR was more intense after fixation with normal-buffered formalin. Equine spinal cord consistently revealed SP-IR, but ChAT-IR was inconsistent. The SP-IR was abolished by prior incubation of SP antibody with SP. Differences were not evident for GFAP and SP staining between recently fixed and stored tissues.

The use of GAR-Cy3 as a second antibody permitted consistent evaluation of slides for $>7$ days. The
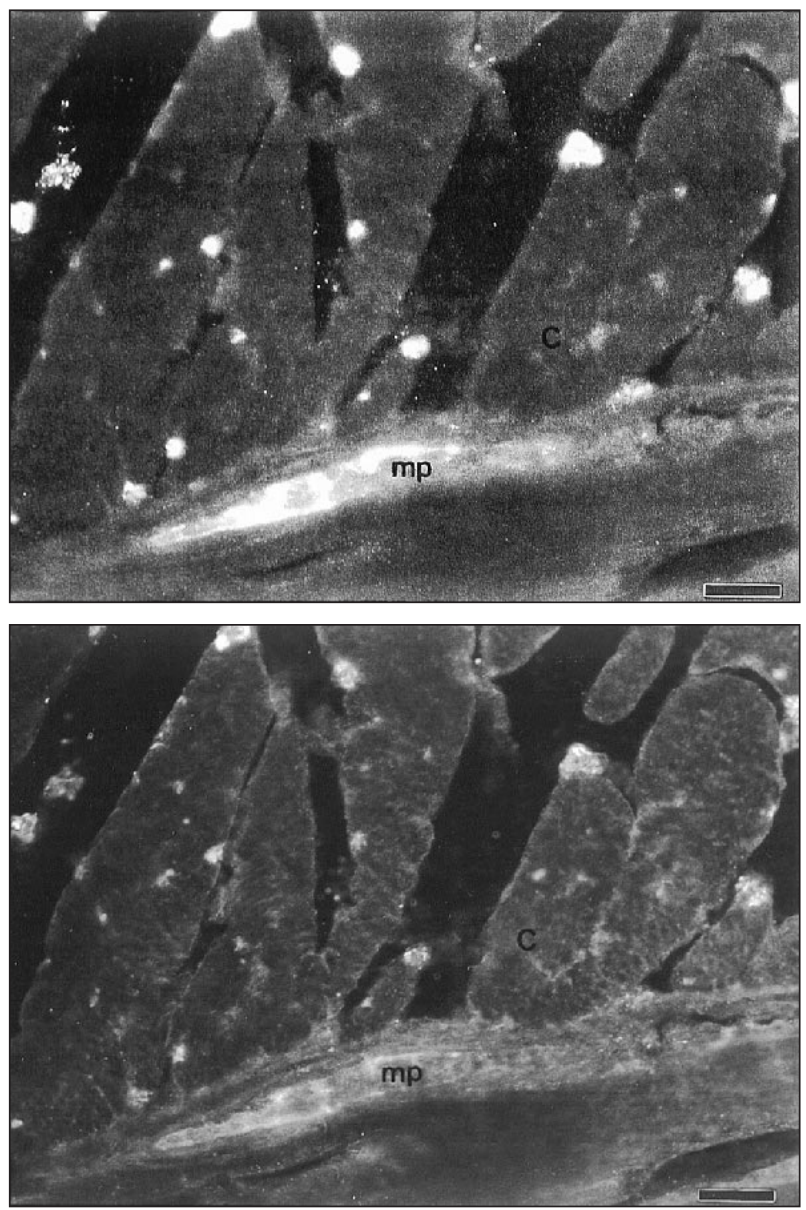

Figure 3-Photomicrographs of a section of jejunum stained with antibodies to SP (top) and choline acetylcholinesterase (ChAT; bottom). Notice the similar staining patterns throughout the myenteric plexus ( $\mathrm{mp}$ ) and circular muscle (C), indicative of colocalization. Tissues were obtained from a clinically normal horse and fixed in Zamboni solution. Bars $=25 \mu \mathrm{m}$.

FITC-labeled slides needed to be evaluated within 2 or 3 days to ensure accurate results, because after that time, the fluorescence faded noticeably.

Immunoreactivity has not been proven to represent authentic ChAT or SP in horses and has been labeled as such (eg, ChAT-like IR). However, for ease of communication, we used the antigen to describe the results (eg, positive for ChAT-IR).

GFAP immunoreactivity-Antibodies to GFAP provided an easily visible outline of the myenteric plexus in all samples examined (Fig 2). Sequential sections provided a reference for other antibodies. 

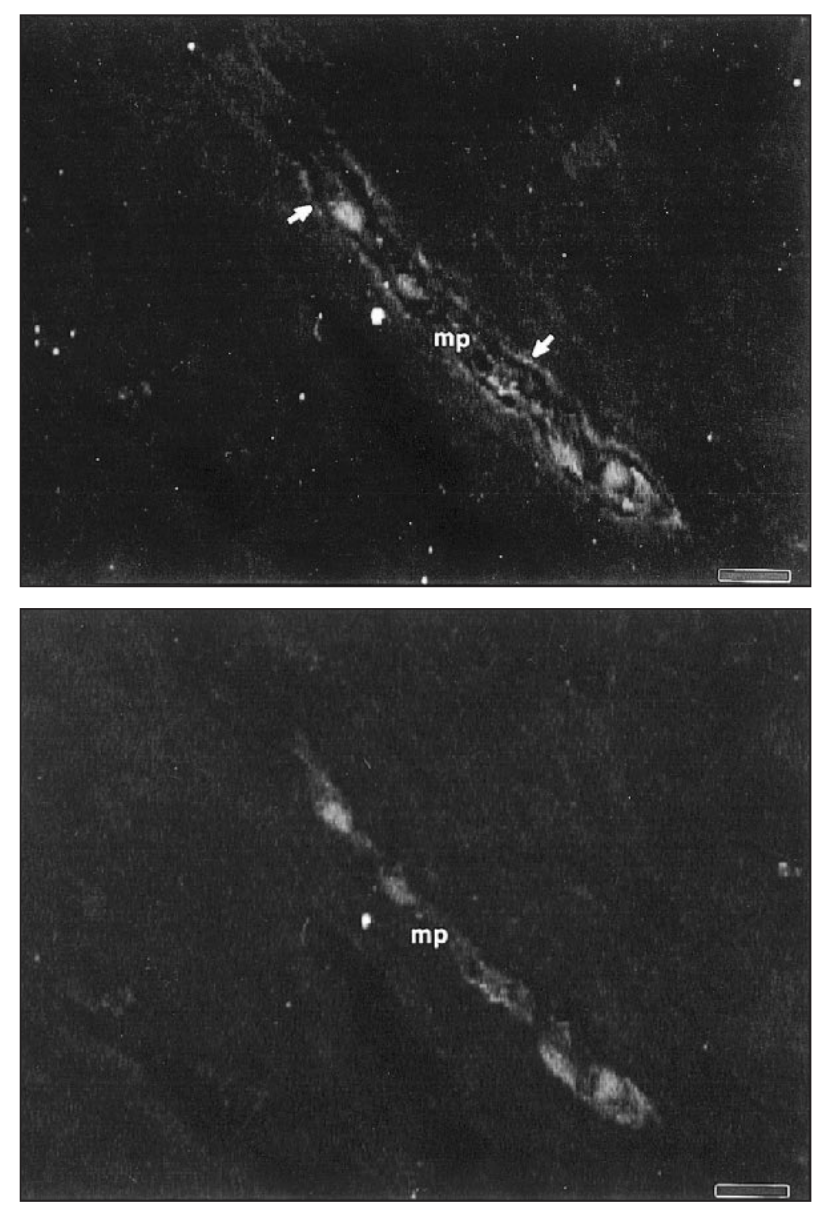

Figure 4-Photomicrographs of a section of jejunum stained with antibodies to SP (top) and ChAT (bottom). Notice the outline of ChAT-containing neurons in the myenteric plexus (mp) with fibers positive for SP (arrows). Tissues were taken obtained from a clinically normal horse and fixed in Zamboni solution. Bars $=50 \mu \mathrm{m}$

SP immunoreactivity-Rabbit and guinea pig anti-SP antibodies positively stained neurons in the myenteric plexus and circular muscle (parallel to the muscle fibers; Fig 3). Areas in the mucosa and submucosa also had positive results for SP-IR. Fetal tissues had a similar pattern.

Double staining with SP and ChAT-Partial colocalization of SP-IR with ChAT-IR was apparent in the myenteric plexus and circular muscle (the range was large but approached $100 \%$ in a few tissues; Fig 3). In other areas of the myenteric plexus, SP-IR neurons were immediately adjacent to ChAT-IR neurons (Fig 4). Many SP-IR areas in the circular muscle did not have evidence of ChAT-IR.

\section{Discussion}

Analysis of results reported here provide additional evidence that SP functions as a neurotransmitter in the equine gastrointestinal tract by documenting the ability of SP to alter smooth muscle contractility, the apparent release of tachykinins from depolarized myenteric neurons, and immunohistochemical localization of SP-like immunoreactivity in the myenteric plexus.
Substance $\mathrm{P}$ contracted smooth muscle of the jejunum, with a greater response in circular muscle than in longitudinal muscle, which is similar to the results seen in other regions of the equine gastrointestinal tract. ${ }^{16}$ This is consistent with immunocytochemical localization of neuronal SP and tachykinin receptors in intestinal smooth muscle..$^{17,26,27}$ In other studies in our laboratory, ${ }^{i}$ tetrodotoxin inhibited a portion of the ileal circular muscle response to SP. Consequently, the effects of SP in horses may be partially mediated by myenteric neurons. Substance P influences the release of other enteric neurotransmitters, including Ach, and Ach may be the final neurotransmitter in the excitatory response to $\mathrm{SP}^{28}$

Addition of CP-96,345 shifted the SP concentration-response curve down and to the right, indicating a noncompetitive inhibition of the effects of SP on muscle strips. The compound CP-96,345 is a nonpeptide NK-1 receptor blocker and was used after a tachykinin-related antagonist in the same preparation did not produce antagonism. ${ }^{29}$ However, equine jejunum is relatively insensitive to CP-96,345; thus, high concentrations of the antagonist are required to inhibit SP activity. ${ }^{3}$ This may reflect a species difference in the NK-1 receptor in horses. ${ }^{30}$ The CP-96,345 has a 10 - to 100-fold higher affinity for the NK-1 receptor in humans, guinea pigs, and rabbits than it does in rats and mice. ${ }^{29}$ At high concentrations, this receptor blocker is capable of interacting with NK-3 receptors and may inhibit NK-3 receptor-mediated Ach release as well as inhibiting the actions of SP at NK-1 receptors. ${ }^{3,31}$ Additionally, CP-96,345 can directly activate smooth muscle contraction via its interaction with Ltype calcium channels. ${ }^{32}$ Evidence supporting the latter hypothesis was infrequent, but increased muscle tension following the addition of CP-96,345 was detected in a few preparations.

Blocking tachykinin receptors with CP-96,345 also altered response of the muscle strips to EFS. This supports the hypothesis that SP or a related tachykinin is released from depolarized myenteric neurons. The inhibitory effects of CP-96,345 on the contractile on response to EFS at higher stimulation intensities would suggest that SP released from myenteric neurons may contract circular and longitudinal muscles, particularly when the muscles are under greater baseline tension. This is consistent with response of the muscles to added SP, but such a response was not observed at low EFS intensities. The ability to detect a SP-induced response only at high stimulation intensities resembles findings in studies on perfused ileum obtained from guinea pigs. ${ }^{33}$ In that study, low distending forces (radial stretch) stimulated peristaltic activity that could be inhibited by blocking the response to Ach. However, the peristaltic activity produced by high distending forces had an atropine-insensitive component that was inhibited by a SP antagonist. Because it is not released until higher amounts of distention, SP may act to elicit fine changes in the reflex pathway, or it may augment cholinergic activity extreme distention or during when there is reduced Ach release. .,34 $^{-34}$

Alterations in EFS-induced off responses in the presence of CP-96,345 suggest that SP can act to relax 
smooth muscle. The NK-1 receptor blocker produced an increase in the off response to EFS in all experiments performed on longitudinal muscle strips and in some experiments performed on already-contracted circular muscle strips. In dogs, SP reportedly has the potential to cause relaxation in the ileum by acting presynaptically on cholinergic neurons, releasing Ach that feeds back to affect muscarinic receptors. ${ }^{9}$ A substantial percentage of neurons containing nitric oxide synthase also express NK-1 receptors in guinea pigs, ${ }^{35}$ and these may be the target of SP-induced inhibition in horses. Physiologically, this type of response may assist in modulating contractile responses to prevent intestinal spasm or complete closure of the lumen.

The apparent combination of SP-induced contraction during EFS followed by SP-induced relaxation could imply a role for SP neurotransmission in pathways regulating intestinal motility. During the preparatory phase of propulsion, the intestine fills with fluid, and intraluminal pressure increases, which causes the longitudinal muscle to contract, thereby widening the lumen. At low filling pressures, this is accompanied by circular muscle relaxation; however, at higher rates of distention, the circular muscle contracts instead. ${ }^{36}$ In other words, substantial distention may stimulate release of SP from neurons, causing contraction of both longitudinal and circular muscle, similar to the effects for the on response in the study reported here. The subsequent relaxation may represent a return of the muscles to a lower baseline tension or may be part of the relaxation components of peristalsis. ${ }^{4}$ Lower-frequency stimulation applied to already contracted circular muscle also resulted in a contractile off response that appeared to be mediated, in part, by SP. This response may represent contraction of circular muscle that is concomitant with relaxation of longitudinal muscle proximal to a bolus of food. ${ }^{4}$

The SP-IR was evident in the myenteric plexus and circular muscle of the jejunum, similar to reports in other mammalian species. ${ }^{26,27}$ The pattern may reflect the location of NK-1 receptors, as was found in the pelvic flexure of horses, ${ }^{17}$ in which case it would correlate with the increased response of circular muscle to SP. Similar to studies in other species, ${ }^{26}$ SP did at least partially colocalize with ChAT. In other instances, SP fibers seemed to surround ChAT-containing neurons, similar to results reported by Cummings et al. ${ }^{11}$ The exact amount of colocalization could not be determined readily because of poor ChAT staining, differing sensitivities of the antibodies to the fixatives, and differing times for peak development among the fluorescent markers. Nonetheless, the association between the 2 antibodies supports our previous findings that release of SP and Ach from neurons may be strongly

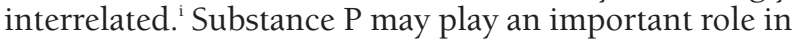
coordinating release of Ach and may help sustain muscle contraction in certain situations, particularly at higher amounts of neuronal stimulation. ${ }^{6,7}$

Substance P (or a related tachykinin) appears to function as a neurotransmitter in the equine jejunum and may act to relax or contract jejunal smooth muscle. The type of response to SP depends on the amount of stimulation, orientation of the muscle, and action of other neurotransmitters. Substance P also may act presynaptically on other neurons to stimulate release of other neurotransmitters. Because of the suspected function attributable to SP for coordinating several groups of neurons, disruption of SP neurotransmission may cause dysfunction of the gastrointestinal tract, and modulation of SP neurotransmission may provide a therapeutic option for motility disorders that are unresponsive to adrenergic and cholinergic agents.

${ }^{a}$ Bladon B, Department of Clinical Veterinary Sciences, School of Clinical Veterinary Sciences, University of Bristol, Bristol, Great Britain: Personal communication, November 1996.

${ }^{b}$ Model S-88 Grass stimulator, Grass Instrument Division, Astro-Med Inc, West Warwick, RI.

'Malone ED. Neural control of equine intestinal motility. $\mathrm{PhD}$ thesis, Department of Clinical and Population Sciences, College of Veterinary Medicine, University of Minnesota, St Paul, 1998.

'Provided by Saul B Kadin, Pfizer Inc, Groton, Conn.

${ }^{e}$ Rabbit anti-glial fibrillary acidic protein antibody, Sigma Biosciences, St Louis, Mo.

${ }^{\mathrm{f}}$ Rabbit anti-substance P antibody, Sigma Biosciences, St Louis, Mo.

${ }^{g}$ Guinea pig anti-substance P antibody, Peninsula Laboratories Inc, Belmont, Calif.

hProvided by Dr R. Elde, Department of Biomedical Sciences, College of Biological Sciences, University of Minnesota, Minneapolis, Minn.

'DeMark-Buik DL. The effects of substance $P$ in the equine cecum in vitro. Science in agriculture thesis. Department of Animal Science, College of Agriculture, Food and Environmental Sciences, University of Minnesota, St Paul, 1999.

\section{References}

1. Malone ED, Kannan MS, Brown DR, et al. Adrenergic, cholinergic, and nonadrenergic-noncholinergic intrinsic innervation of the equine jejunum in horses. Am J Vet Res 1999;60:898-904.

2. Hoyle CHV, Burnstock G. Criteria for defining enteric neurotransmitters. In: Gaginella TS, ed. Methods in gastrointestinal pharmacology. New York: CRC Press, 1996;123-140.

3. Mussap CJ, Geraghty DP, Burcher E. Tachykinin receptors: a radioligand binding perspective. J Neurochem 1993;60:1987-2009.

4. Guilford WG. The enteric nervous system: function, dysfunction, and pharmacological manipulation. Semin Vet Med Surg (Small Anim) 1990;5:46-56.

5. Brown DR, Parsons AM, O'Grady SM. Substance P produces sodium and bicarbonate secretion in porcine jejunal mucosa through an action on enteric neurons. J Pharmacol Exp Ther 1992;261: $1206-1212$.

6. Costa M, Furness JB, Pullin CO, et al. Substance P enteric neurons mediate non-cholinergic transmission to the circular muscle of the guinea-pig intestine. Naunyn Schmeideberg Arch Pharmacol 1985;328:446-453.

7. Grider JR. Identification of neurotransmitters regulating intestinal peristaltic reflex in humans. Gastroenterology 1989;97: $1414-1419$.

8. Rothstein RD, DeRiso J, Ouyang A. Ileocecal sphincter contraction to colonic distension: a tachykinin-mediated spinal reflex. Am J Physiol 1990;258:G585-G590.

9. Fox JET, Daniel EE, MacDonald TJ, et al. Evidence for a muscarinic inhibitory brake activated by peptides in the canine small intestine. In: Roman C, ed. International symposium on gastrointestinal motility. Lancaster, UK: MTP Press Ltd, 1984;327-333.

10. Bishop AE, Hodson NP, Major JH, et al. The regulatory peptide system of the large bowel in equine grass sickness. Experientia 1984;40:801-806.

11. Cummings JF, Sellers AF, Lowe JE. Distribution of substance P-like immunoreactivity in the enteric neurons of the large colon of normal and amitraz-treated ponies: an immunocytochemical study. Equine Vet J 1984;17:23-39.

12. Kitamura N, Yamada J, Calingasan NY, et al. Immunocytochemical distribution of endocrine cells in the gastrointestinal tract of the horse. Equine Vet J 1984;16:103-107. 
13. Burns GA, Cummings JF. Neuropeptide distributions in the colon, cecum and jejunum of the horse. Anat Rec 1993;236:341-350.

14. Pearson GT. Structural organization and neuropeptide distributions in the equine enteric nervous system: an immunohistochemical study using whole-mount preparations from the small intestine. Cell Tissue Res 1994;276:523-534.

15. Sellers AF, Lowe JE, Cummings JF. Trials of serotonin, substance $\mathrm{P}$ and $\alpha_{2}$-adrenergic receptor effects on the equine large colon Cornell Vet 1985;75:319-323.

16. Belloli C, Arioli F, Beretta C, et al. In vitro effects of tachykinins on the smooth musculature of horse gut. J Vet Pharmacol Ther 1994;17:379-383.

17. Sonea IM, Wilson DV, Bowker RM, et al. Tachykinin receptors in the equine pelvic flexure. Equine Vet J 1997;29:306-312.

18. Goldin E, Karmeli F, Selinger Z, et al. Colonic substance P levels are increased in ulcerative colitis and decreased in chronic severe constipation. Dig Dis Sci 1989;34:754-757.

19. Mazumdar S, Das KM. Immunocytochemical localization of vasoactive intestinal peptide and substance $\mathrm{P}$ in the colon from normal subjects and patients with inflammatory bowel disease. Am J Gastroenterol 1992;87:176-181.

20. Palmer JM, Greenwood B. Regional content of enteric substance $\mathrm{P}$ and vasoactive intestinal peptide during intestinal inflammation in the parasitized ferret. Neuropeptides 1993;25:95-103.

21. Agro A, Stanisz AM. Inhibition of murine intestinal inflammation by anti-substance P antibody. Reg Immunol 1993;5:120-126.

22. Moore RM, Charalambous AC, Masty J. Alterations in colonic arterial and venous plasma neuropeptide concentrations in horses during low-flow ischemia and reperfusion of the large colon Am J Vet Res 1996;57:1200-1205.

23. Bancroft JD, Stevens A. Fixation and fixatives. In: Bancroft JD, Stevens A, eds. Theory and practice of histological techniques. 3rd ed. New York: Churchill Livingstone Inc, 1990;33-34.

24. Krammer HJ, Kuhnel W. Immunohistochemistry for intermediate filaments in the enteric nervous system of the porcine small intestine. Anat Anz 1992;174:275-278.

25. Schemann M, Sann H, Schaaf C, et al. Identification of cholinergic neurons in enteric nervous system by antibodies against choline acetyltransferase. Am J Physiol 1993;265:G1005-G1009.

26. Steele PA, Brookes SJH, Costa M. Immunohistochemical identification of cholinergic neurons in the myenteric plexus of guinea-pig small intestine. Neuroscience 1991;45:227-239.

27. Costa M, Brookes SJH, Waterman S, et al. Enteric neuronal circuitry and transmitters controlling intestinal motor function. In: Holle GE, ed. Advances in the innervation of the gastrointestinal tract. Amsterdam: Elsevier Science Publishers, 1992;115-121.

28. Yau WM, Youther ML. Direct evidence for a release of acetylcholine from the myenteric plexus of guinea-pig small intestine by substance P. Eur J Pharmacol 1982;81:665-668.

29. Snider RM, Constantine JW, Lowe JA III, et al. A potent nonpeptide antagonist of the substance $\mathrm{P}\left(\mathrm{NK}_{1}\right)$ receptor. Science 1991;251:435-437.

30. Hall JM, Caulfield MP, Watson SP, et al. Receptor subtypes or species homologues: relevance to drug discovery. Trends Pharmacol Sci 1993;14:376-383.

31. Presti ME, Gardner JD. Receptor antagonists for gastrointestinal peptides. Am J Physiol 1993;264:G399-G406.

32. Guard S, Boyle SJ, Tang K-W et al. The interaction of the $\mathrm{NK}_{1}$ receptor antagonist CP-96,345 with L-type calcium channels and its functional consequence. Br J Pharmacol 1993;110: 385-391.

33. Barthó L, Holzer P, Donnerer J, et al. Evidence for the involvement of substance $\mathrm{P}$ in the atropine-resistant peristalsis of the guinea-pig ileum. Neurosci Lett 1982;32:69-74.

34. Wechsung E, Houvenaghel A. Influence of substance P on gastrointestinal myoelectrical activity in the conscious piglet. Zentralbl Veterinarmed A 1992;39:561-567.

35. Portbury AL, Furness JB, Young HM, et al. Localisation of NKl receptor immunoreactivity to neurons and interstitial cells of the guinea-pig gastrointestinal tract. I Comp Neurol 1996;367: 342-351.

36. Waterman SA, Costa M, Tonini M. Accommodation mediated by enteric inhibitory reflexes in the isolated guinea-pig small intestine. J Physiol (Lond) 1994;474:539-546. 